\title{
DISCOVERY OF A COMPANION AT THE L/T TRANSITION WITH THE WIDE-FIELD INFRARED SURVEY EXPLORER
}

\author{
N. P. Loutrel ${ }^{1}$, K. L. LuHMAN ${ }^{1,2}$, P. J. LOWRANCE ${ }^{3}$, AND J. J. BochanSKi ${ }^{1}$ \\ ${ }^{1}$ Department of Astronomy and Astrophysics, The Pennsylvania State University, University Park, PA 16802, USA; loutrel@ astro.psu.edu \\ ${ }^{2}$ Center for Exoplanets and Habitable Worlds, The Pennsylvania State University, University Park, PA 16802, USA \\ ${ }^{3}$ Infrared Processing and Analysis Center, California Institute of Technology 100-22, Pasadena, CA 91125, USA \\ Received 2011 June 14; accepted 2011 July 9; published 2011 September 12
}

\begin{abstract}
We report the discovery of a substellar companion to the nearby solar-type star HD 46588 (F7V, 17.9 pc, $\tau \sim 3 \mathrm{Gyr}$ ). HD $46588 \mathrm{~B}$ was found through a survey for common proper motion companions to nearby stars using data from the Wide-field Infrared Survey Explorer (WISE) and the Two Micron All Sky Survey (2MASS). It has an angular separation of 79'.2 from its primary, which corresponds to a projected physical separation of 1420 AU. We have measured a spectral type of L9 for this object based on near-infrared spectroscopy performed with TripleSpec at Palomar Observatory. We estimate a mass of $0.064_{-0.019}^{+0.008} M_{\odot}$ from a comparison of its luminosity to the values predicted by theoretical evolutionary models for the age of the primary. Because of its companionship to a wellstudied star, HD $46588 \mathrm{~B}$ is one of the few known brown dwarfs at the L/T transition for which both age and distance estimates are available. Thus, it offers new constraints on the properties of brown dwarfs during this brief evolutionary phase. The discovery of HD $46588 \mathrm{~B}$ also illustrates the value of the WISE for identifying brown dwarfs in the solar neighborhood via their proper motions.
\end{abstract}

Key words: binaries: visual - brown dwarfs - infrared: stars - proper motions - stars: individual (HD 46588, 2MASS J06462756+7935045, WISEP J064627.10+793457.8)

Online-only material: color figure

\section{INTRODUCTION}

$\mathrm{L}$ and $\mathrm{T}$ dwarf companions to nearby stars are valuable for studying the structure and evolution of brown dwarfs as well as their origin. Because the ages and metallicities of these companions often can be determined via their primaries, they can be used to calibrate atmospheric and evolutionary models (Kirkpatrick et al. 2005; Cushing et al. 2008; Helling et al. 2008; Burgasser et al. 2010b). Meanwhile, theories for the formation of brown dwarfs can be tested with measurements of the fraction of stars that harbor wide low-mass companions and the frequency of tight binaries among these companions (Reipurth \& Clarke 2001; Bate et al. 2003; Stamatellos \& Whitworth 2009).

Multiple strategies have been employed in identifying cool companions to stars in the solar neighborhood. One approach is to define a sample of stars and search for companions to them (Becklin \& Zuckerman 1988; Nakajima et al. 1995; Potter et al. 2002; Lowrance et al. 2005; Biller et al. 2006; Metchev \& Hillenbrand 2006; Mugrauer et al. 2006; Luhman et al. 2007, 2011). Alternatively, one can begin with a sample of known brown dwarfs and check whether they are companions to stars (Burgasser et al. 2000; Kirkpatrick et al. 2001; Wilson et al. 2001; Scholz et al. 2003; Burningham et al. 2009; Faherty et al. 2010, 2011; Goldman et al. 2010; Scholz 2010; Zhang et al. 2010; Day-Jones et al. 2011). Late-type companions also can be uncovered through a search for pairs of comoving objects across a large area of sky (Radigan et al. 2008). Proper motion measurements are essential in all of these strategies, either for the initial identification of candidate companions or for confirming candidates that were selected through photometry. These proper motions can be measured through dedicated imaging or archival data from wide-field surveys like the Two Micron All Sky Survey (2MASS; Skrutskie et al. 2006), the Sloan Digital Sky Survey (SDSS; York et al. 2000), and the United Kingdom Infrared Telescope Infrared Deep Sky Survey (UKIDSS; Lawrence et al. 2007). A new source of astrometry has recently become available via the Wide-field Infrared Survey Explorer (WISE; Wright et al. 2010). This mission has performed the first all-sky survey at infrared (IR) wavelengths since 2MASS, thus providing an opportunity to measure proper motions for 2MASS sources across the entire sky. Indeed, proper motions incorporating WISE data have already been exploited for searching for free-floating $L$ and T dwarfs (Scholz et al. 2011; Gizis et al. 2011).

We have begun a search for faint common proper motion companions to nearby stars using astrometry from 2MASS and WISE. In this paper, we report the discovery of a substellar companion to the solar-type star HD 46588. We describe the methods that uncovered this object as a possible companion (Section 2) and present spectroscopy that confirms its cool nature (Section 3). We then examine the physical properties of this new companion (Section 4) and discuss its relevance to studies of L and T dwarfs (Section 5).

\section{2MASS-WISE PROPER MOTION SURVEY}

Between 1997 and 2001, the 2MASS telescopes collected images of the entire sky in broadband filters centered at 1.25 , 1.65 , and $2.16 \mu \mathrm{m}\left(J, H, K_{s}\right.$; Skrutskie et al. 2006). In 2010, the WISE satellite mapped the sky at 3.4, 4.6, 12, and $22 \mu \mathrm{m}$ (W1-W4; Wright et al. 2010). The typical magnitudes that correspond to signal-to-noise ratio $(\mathrm{S} / \mathrm{N})=5$ for $2 \mathrm{MASS}$ and WISE are $J=16.5, H=15.8, K_{s}=15.0, W 1=16.5$, $W 2=15.5, W 3=11.2$, and $W 4=7.9$ in unconfused areas of sky and (for WISE) in the ecliptic. WISE achieves better sensitivity at higher ecliptic latitudes because of greater depth of coverage. Given these photometric limits and the near- and mid-IR colors of L and T dwarfs (Leggett et al. 2010), a survey 

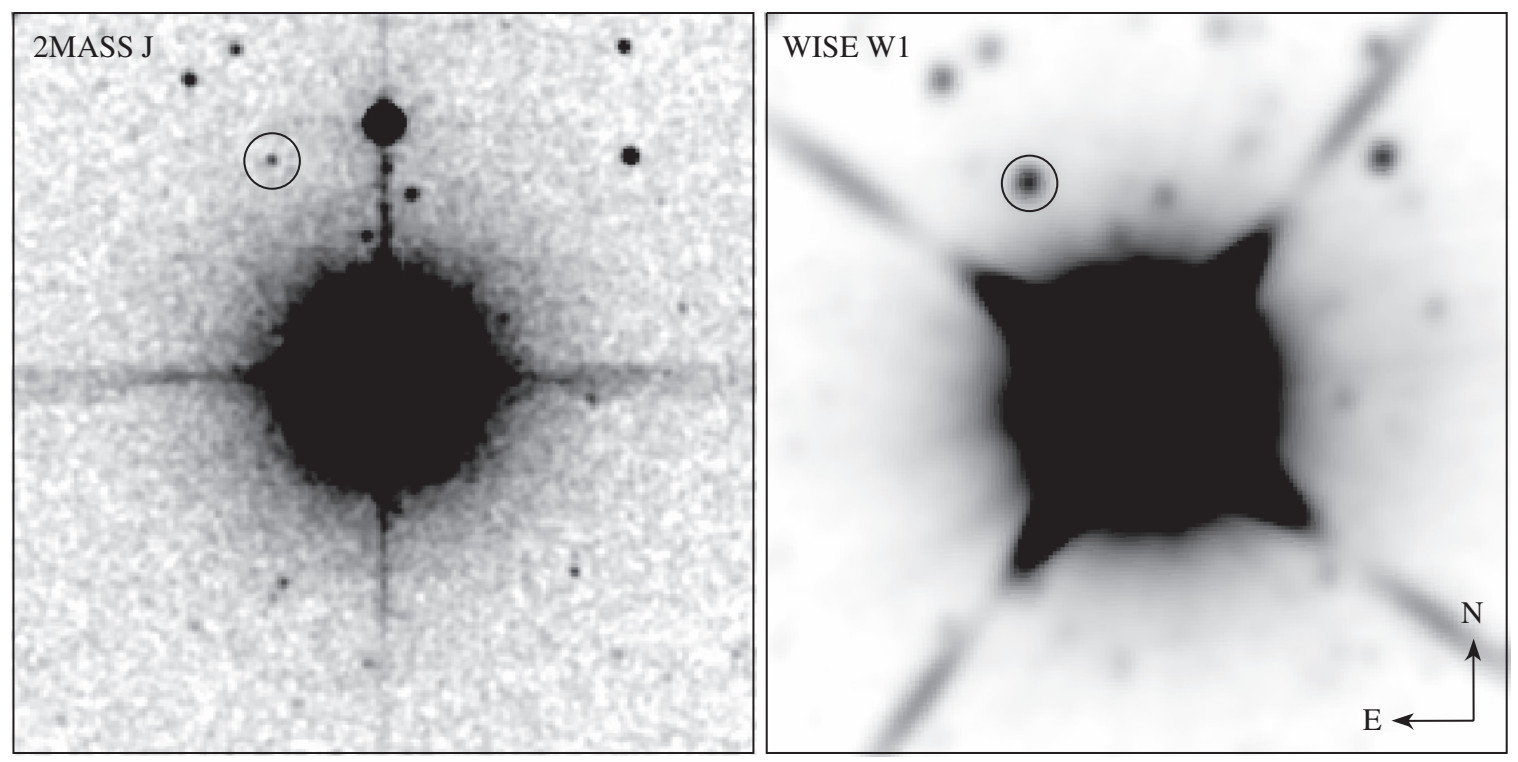

Figure 1. 2MASS and WISE images of HD $46588 \mathrm{~A}$ and B. The size of each image is $4^{\prime} \times 4^{\prime}$.

that requires detections in both $2 \mathrm{MASS}$ and WISE will be limited by the sensitivity of 2 MASS at $J$.

The astrometric precision of $2 \mathrm{MASS}$ is slightly less than 0 .' 1 for $\mathrm{S} / \mathrm{N}>10$ and approaches 0.2 at the detection limit (Skrutskie et al. 2006). For WISE, the precision is 0.2 for brighter sources $(W 1<13)$ and approaches $1^{\prime \prime}$ in declination for some of the fainter objects because of an error in the preliminary data processing. Given the elapsed time of $\sim 10$ years between 2MASS and WISE, proper motions measured from their astrometry should have accuracies of $\lesssim 10 \%$ at brighter magnitudes for $\mu \gtrsim 0.2 \mathrm{yr}^{-1}$. Therefore, we have considered for our survey all known main-sequence stars, white dwarfs, and brown dwarfs in the solar neighborhood with proper motions exceeding this threshold (e.g., Subasavage et al. 2005; Lepine 2005, 2008; Faherty et al. 2009).

To search for possible late-type companions to a given primary, we begin by retrieving lists of coordinates and photometry for all objects within a radius of $1^{\circ}$ that appear in the 2MASS Point Source Catalog and the WISE Preliminary Release Source Catalog. We match the two lists to identify pairs of 2MASS and WISE sources whose astrometric offsets correspond to the expected motion of the primary based on its known proper motion. To be considered a candidate companion, we also require that an object's colors and magnitudes (or limits) from 2MASS and WISE are consistent with those of $\mathrm{M}, \mathrm{L}$, and $\mathrm{T}$ dwarfs at the distance of the primary. To characterize the colors of the latter, we retrieved photometry from the 2MASS and WISE catalogs for late-type dwarfs that have known distances (Leggett et al. 2010, and references therein). This process typically produces $\lesssim 2$ candidate companions for most primaries. Stars that have low galactic latitudes or small proper motions can have dozens of candidates. We then visually inspect the 2MASS and WISE images to identify and reject false candidates, which often consist of one component of a resolved pair of 2MASS sources matched to an unresolved blend of the pair in WISE.

Through our analysis of the 2MASS and WISE data for nearby stars, we have identified a new companion to the F7V star HD 46588. The companion is designated as 2MASS J06462756+7935045 and WISEP J064627.10+793457.8, and we refer to it as HD $46588 \mathrm{~B}$. It has a projected separation of

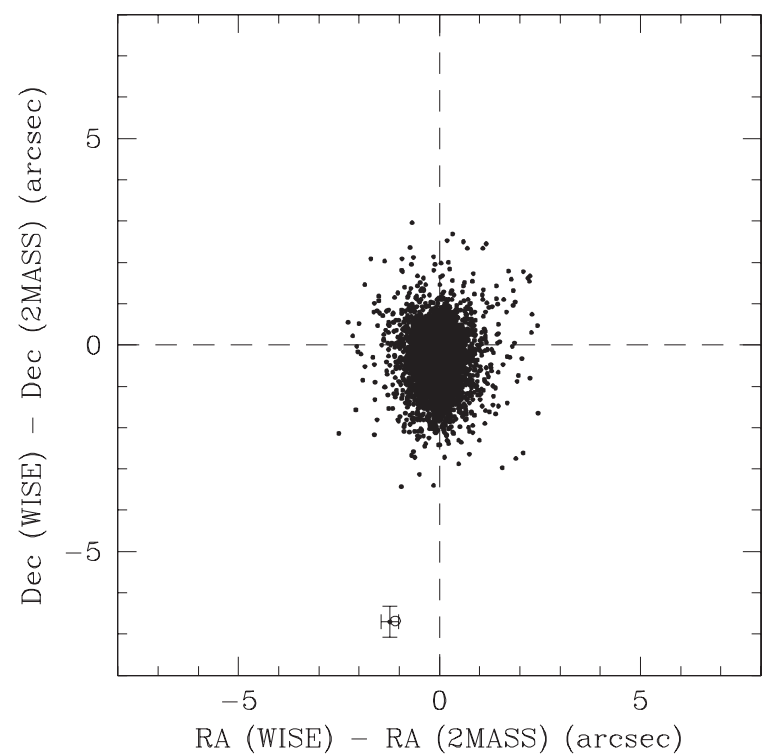

Figure 2. Differences in coordinates of sources within $1^{\circ}$ of HD 46588 between 2MASS and WISE (points). The motion of one of these sources ( $1 \sigma$ error bars) is consistent with the expected motion of HD 46588 (circle). The vertical elongation in the distribution of offsets is a reflection of the systematically larger uncertainties in the preliminary measurements of declination for faint sources from WISE (Section 2).

$\sim 80^{\prime \prime}$, corresponding to $1420 \mathrm{AU}$ at the distance of the primary (17.9 pc; van Leeuwen 2007). We show 2MASS and WISE images of the pair in Figure 1. The agreement between the motions of HD $46588 \mathrm{~A}$ and B is demonstrated in Figure 2, where we plot the differences in equatorial coordinates between 2MASS and WISE for all stars within a radius of $1^{\circ}$ and with $W 2$ uncertainties less than $0.1 \mathrm{mag}$. For stars that have $W 2$ fluxes within \pm 0.5 mag of HD $46588 \mathrm{~B}$, the standard deviations of the differences in right ascension and declination are $00^{\prime \prime} 21$ and $0^{\prime \prime} .37$, respectively. The colors and magnitudes of HD $46588 \mathrm{~B}$ from 2MASS and WISE are consistent with those of a cool dwarf at the distance of the primary, as illustrated in the color-magnitude diagrams in Figure 3. The colors of HD 46588 B suggest that it is near the boundary between $\mathrm{L}$ and $\mathrm{T}$ dwarfs. 

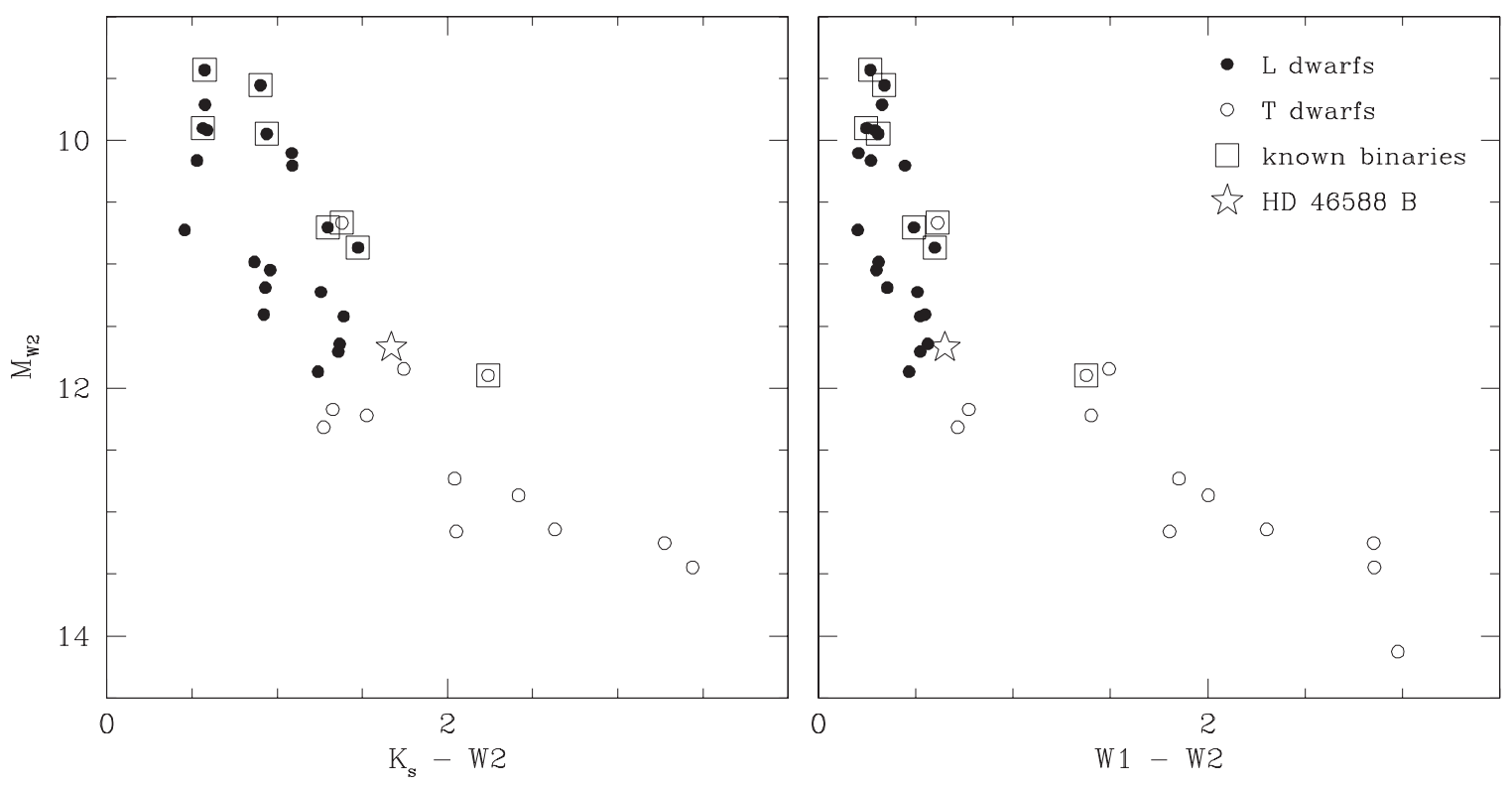

Figure 3. $M_{W 2}$ vs. $K_{s}-W 2$ and $M_{W 2}$ vs. $W 1-W 2$ for HD 46588 B (star) and $\mathrm{L}$ and T dwarfs that have known distances (Leggett et al. 2010, and references therein) and that have WISE and $K_{S}$ (or transformed $K_{\mathrm{MKO}}$ ) measurements available (filled and open circles).

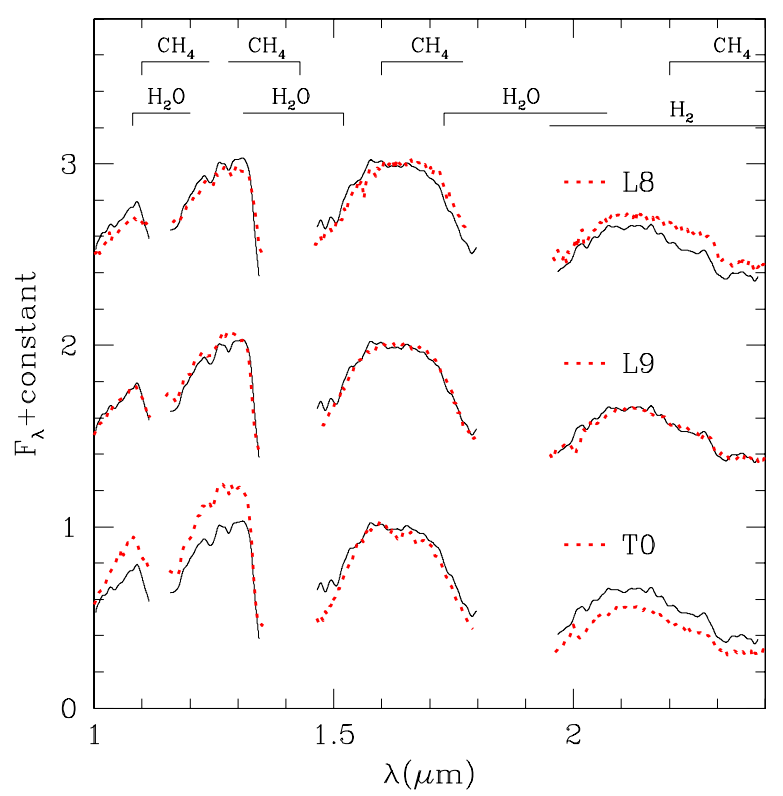

Figure 4. Spectrum of HD 46588 B (solid line) compared to data for the near-IR standards 2MASSW J1632291+190441 (L8; Burgasser et al. 2004), DENIS-P J0255-4700 (L9; Burgasser et al. 2006a), and SDSS J120747.17+024424.8 (T0; Looper et al. 2007). We classify HD 46588 B as L9 based on this comparison. Each spectrum is normalized at its maximum flux in the $H$ band.

(A color version of this figure is available in the online journal.)

\section{SPECTROSCOPY}

To confirm its cool nature, we obtained a near-IR spectrum of HD 46588 B with the TripleSpec spectrograph (Herter et al. 2008) on the Palomar $5 \mathrm{~m}$ Hale telescope on the night of 2011 May 12. TripleSpec produces spectra that extend from $1-2.4 \mu \mathrm{m}$ and have a resolution of 2700 . We collected eight 5 min exposures of HD $46588 \mathrm{~B}$ in an ABBA dither pattern along the slit $\left(1^{\prime \prime} \times 30^{\prime \prime}\right)$. We also observed HD 45560 (A1V) to provide telluric correction. The slit was aligned to the parallactic angle for these observations. We reduced the data with a version of the Spextool package (Cushing et al. 2004) that has been modified
Table 1

Properties of HD 46588 A and B

\begin{tabular}{|c|c|c|}
\hline Parameter & Value & Reference \\
\hline \multicolumn{3}{|c|}{ HD 46588 A } \\
\hline Distance & $17.87 \pm 0.05 \mathrm{pc}$ & 1 \\
\hline$\mu_{\alpha}$ & $-99.16 \pm 0.19$ mas yr $^{-1}$ & 1 \\
\hline$\mu_{\delta}$ & $-603.76 \pm 0.26$ mas yr $^{-1}$ & 1 \\
\hline Spectral Type & F7V & 2 \\
\hline Age & $2.8 \pm 1.5 \mathrm{Gyr}$ & 3 \\
\hline$[\mathrm{Fe} / \mathrm{H}]$ & -0.21 & 3 \\
\hline \multicolumn{3}{|c|}{ HD $46588 \mathrm{~B}$} \\
\hline Separation & $79^{\prime \prime} .2 \pm 0.2$ (1420 AU) & 4 \\
\hline Position Angle & $27.46 \pm 0.15$ & 4 \\
\hline$\mu_{\alpha}$ & $-112 \pm 19$ mas yr$^{-1}$ & 4,5 \\
\hline$\mu_{\delta}$ & $-606 \pm 33$ mas yr $^{-1}$ & 4,5 \\
\hline$J$ & $16.26 \pm 0.09 \mathrm{mag}$ & 4 \\
\hline$H$ & $15.08 \pm 0.07 \mathrm{mag}$ & 4 \\
\hline$K_{s}$ & $14.60 \pm 0.09 \mathrm{mag}$ & 4 \\
\hline$W 1$ & $13.58 \pm 0.03 \mathrm{mag}$ & 5 \\
\hline$W 2$ & $12.93 \pm 0.03 \mathrm{mag}$ & 5 \\
\hline Spectral Type & $\mathrm{L} 9 \pm 1$ & 6 \\
\hline $\log L / L_{\odot}$ & $-4.68 \pm 0.05$ & 6 \\
\hline$T_{\text {eff }}$ & $1360_{-80}^{+50} \mathrm{~K}$ & 6 \\
\hline Mass & $0.064_{-0.019}^{+0.008} M_{\odot}$ & 6 \\
\hline
\end{tabular}

References. (1) van Leeuwen 2007; (2) Abt 2009; (3) Casagrande et al. 2011; (4) 2MASS Point Source Catalog; (5) WISE Preliminary Release Source Catalog; (6) this work.

for use with data from TripleSpec (M. Cushing 2011, private communication). This software corrects for telluric absorption with the methods described by Vacca et al. (2003).

We present the reduced spectrum of HD 46588 B in Figure 4. It exhibits strong absorption bands from $\mathrm{H}_{2} \mathrm{O}$, which confirms that it is a cool object. To measure its spectral type, we compared the spectrum of HD 46588 B to data from the SpeX Prism Spectral Libraries ${ }^{4}$ for $\mathrm{L}$ and $\mathrm{T}$ dwarf standards (Burgasser et al. 2006a). Although the metallicity of HD 46588 appears

\footnotetext{
4 http://www.browndwarfs.org/spexprism
} 


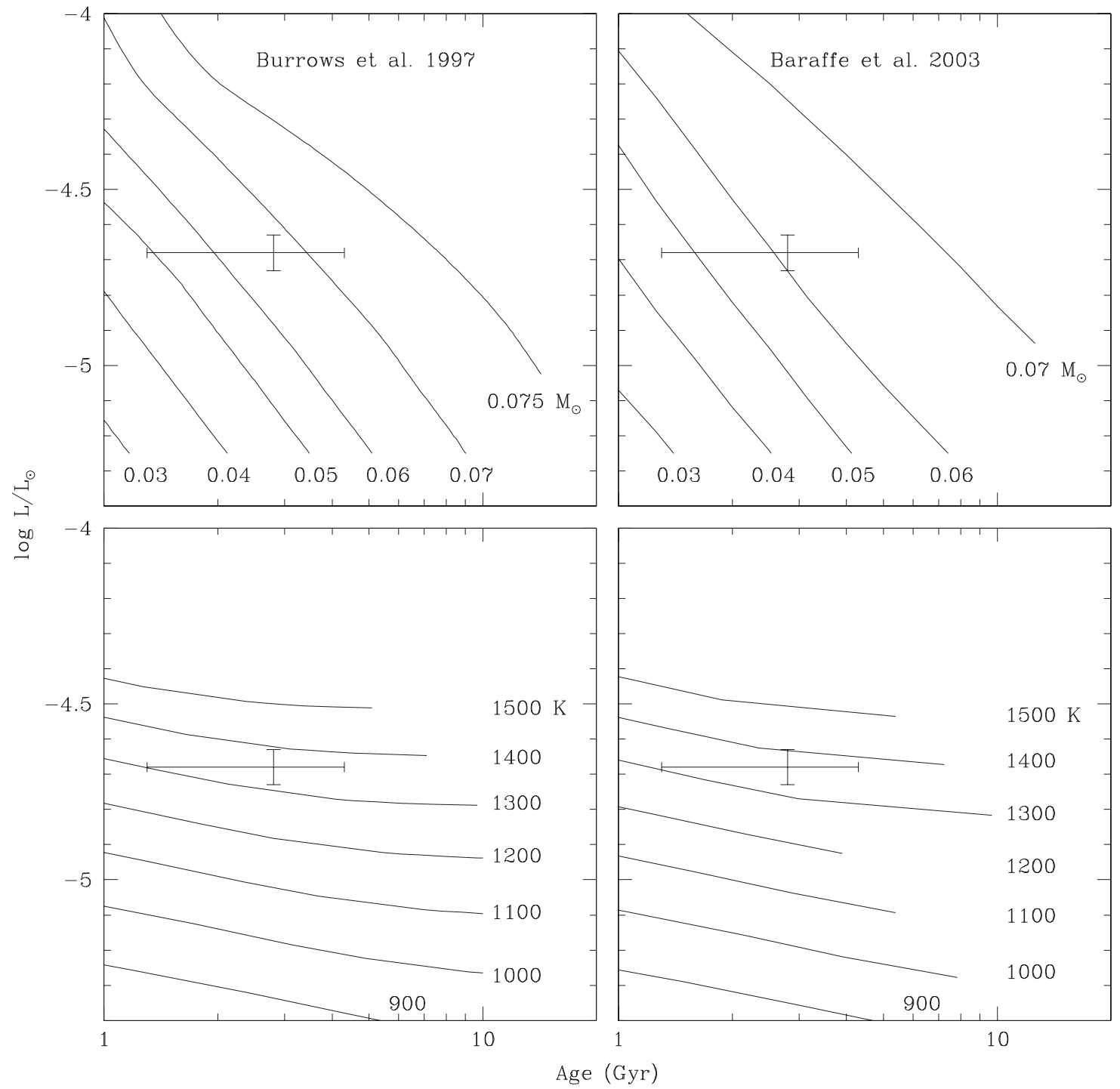

Figure 5. Luminosity of HD 46588 B compared to the luminosities as a function of age predicted by the theoretical evolutionary models of Burrows et al. (1997) and Baraffe et al. (2003) for constant values of mass and temperature.

to be subsolar $([\mathrm{Fe} / \mathrm{H}]=-0.21$; Casagrande et al. 2011), it is only $\sim 0.1$ dex below the mean value for stars in the solar neighborhood (Nordström et al. 2004; Holmberg et al. 2007). Thus, normal dwarf standards should be suitable for the classification of HD 46588 B. For comparison to the standards, the spectrum of HD $46588 \mathrm{~B}$ was smoothed to the same resolution as the SpeX data $(R \sim 100)$. We find that HD 46588 B closely resembles standards with a near-IR spectral type of L9, as illustrated in Figure 4. This classification is consistent with that expected based on its photometry (Figure 3).

\section{PHYSICAL PROPERTIES}

Our proper motion measurements and spectroscopy have demonstrated the companionship of HD 46588 B. Therefore, it should have the same distance, age, and metallicity as its primary. In Table 1, we list previous measurements and estimates of these parameters for HD 46588. To estimate the bolometric luminosity of HD $46588 \mathrm{~B}$, we converted its 2MASS $K_{s}$ magnitude to the Mauna Kea Observatory (MKO) photometric system (Stephens \& Leggett 2004) and applied a bolometric correction of $\mathrm{BC}_{K}=3.1$ (Golimowski et al. 2004), the distance of the primary, and the absolute bolometric magnitude for the Sun $\left(M_{\mathrm{bol} \odot}=4.75\right)$. We arrive at a value of $\log L / L_{\odot}=-4.68 \pm 0.05$. Based on this luminosity and the age of the primary, we estimate masses of $0.067_{-0.017}^{+0.005} M_{\odot}$ and $0.061_{-0.016}^{+0.005} M_{\odot}$ from the evolutionary models of Burrows et al. (1997) and Baraffe et al. (2003), respectively (see Figure 5). Both of these estimates are fully below the hydrogen burning mass limit $\left(\sim 0.075 M_{\odot}\right.$; Chabrier \& Baraffe 2000; Burrows et al. 1997), indicating that HD 46588 B is probably a brown dwarf. To encompass both ranges of masses, we adopt a mass of $0.064_{-0.019}^{+0.008} M_{\odot}$. These models imply an effective temperature of $1360_{-80}^{+50} \mathrm{~K}$, which agrees with previous estimates for late L dwarfs (Golimowski et al. 2004; Vrba et al. 2004). The photometric, astrometric, and physical properties of HD 46588 B are summarized in Table 1. We note that the WISE Preliminary Release Source Catalog contains an additional photometric measurement in $W 3$, but the detection is marginal. The companion is not detected in $W 4$.

\section{DISCUSSION}

We have presented the discovery of a widely separated L9 companion to the nearby F7V star HD 46588. Among 
previously known companions, G1584 C most closely resembles HD $46588 \mathrm{~B}$ in terms of the spectral types of the system components (G1/G3/L8; Kirkpatrick et al. 2001). Other lateL/early-T companions to stars include G1337 CD (T0; Wilson et al. 2001; Burgasser et al. 2005), HD 203030 B (L7.5; Metchev \& Hillenbrand 2006), HN Peg B (T2.5; Luhman et al. 2007), and LHS 2397aB (L7; Dupuy et al. 2009).

Brown dwarfs near the $\mathrm{L} / \mathrm{T}$ transition have served as laboratories for studying the complex atmospheric changes that occur between L and T dwarfs (Burgasser et al. 2010a). Observations of binaries have played a central role in these studies. For instance, systems with spectral types of late L or early $\mathrm{T}$ appear to have a high binary fraction compared to other types (Burgasser et al. 2006b; Liu et al. 2006). This has been explained by rapid evolution of brown dwarfs through the $\mathrm{L} / \mathrm{T}$ transition, which causes single transition objects to be rare and binaries containing earlier and later types to dominate systems that have composite types near the $\mathrm{L} / \mathrm{T}$ boundary (Burgasser et al. 2006b; Burgasser 2007). These binaries often appear overluminous relative to other brown dwarfs at the same color or spectral type. HD $46588 \mathrm{~B}$ is somewhat overluminous in $M_{W 2}$ versus $K_{s}-W 2$ but appears near the lower envelope of the L/T dwarf sequence in $M_{W 2}$ versus $W 1-W 2$ (Figure 3 ). Thus, it does not show clear evidence of binarity. Regardless of whether it is single or binary, since its distance is known, HD $46588 \mathrm{~B}$ offers an opportunity to better constrain the relations between spectral type and absolute magnitudes (e.g., $M_{\text {bol }}, M_{K}$ ) near the $\mathrm{L} / \mathrm{T}$ transition, which influence the modeling of binary statistics and surface densities of $\mathrm{L} / \mathrm{T}$ objects as well as the identification of overluminous systems (Burgasser 2007). In addition, because of its companionship to a star, HD $46588 \mathrm{~B}$ has the best available age estimate of any known object at a spectral type of L9. By combining its age and luminosity with evolutionary models, we have been able to estimate its temperature, and thus constrain the temperature of the $\mathrm{L} / \mathrm{T}$ boundary. The available measurements of $M_{\mathrm{bol}}, M_{K}$, and temperature for HD $46588 \mathrm{~B}$ are consistent with those derived previously for companions with ages of $>1$ Gyr (Kirkpatrick et al. 2001; Dupuy et al. 2009), but these relations could be more tightly constrained by improving the accuracy of near-IR photometry for HD $46588 \mathrm{~B}$.

Data from the WISE mission have been recently used to identify new free-floating late-type members of the solar neighborhood based on their colors (Mainzer et al. 2011; Burgasser et al. 2011; Cushing et al. 2011; Kirkpatrick et al. 2011) and proper motions (Scholz et al. 2011; Gizis et al. 2011). Our discovery of HD 46588 B illustrates the added value of WISE for uncovering cool companions to nearby stars via their proper motions. This object also helps to complete the census of $\mathrm{L}$ dwarfs within 20 pc from the Sun (Cruz et al. 2007).

We thank Michael Cushing for assistance with reducing the TripleSpec data and Sandy Leggett for her helpful referee report. K. L., N. L., and J. B. acknowledge support from grant AST-0544588 from the National Science Foundation. This publication makes use of data products from the following resources: the Wide-field Infrared Survey Explorer, which is a joint project of the University of California, Los Angeles, and the Jet Propulsion Laboratory/California Institute of Technology, funded by the National Aeronautics and Space Administration; the NASA/IPAC Infrared Science Archive, which is operated by the Jet Propulsion Laboratory, California Institute of Technology, under contract with the National Aeronautics and Space Administration; the SpeX
Prism Spectral Libraries, maintained by Adam Burgasser at http://www.browndwarfs.org/spexprism; the M, L, and T dwarf compendium housed at http://DwarfArchives.org and maintained by Chris Gelino, Davy Kirkpatrick, and Adam Burgasser. The Center for Exoplanets and Habitable Worlds is supported by the Pennsylvania State University, the Eberly College of Science, and the Pennsylvania Space Grant Consortium.

\section{REFERENCES}

Abt, H. A. 2009, ApJS, 180, 117

Baraffe, I., Chabrier, G., Barman, T. S., Allard, F., \& Hauschildt, P. H. 2003, A\&A, 402, 701

Bate, M. R., Bonnell, I. A., \& Bromm, V. 2003, MNRAS, 339, 577

Becklin, E. E., \& Zuckerman, B. 1988, Nature, 336, 656

Biller, B. A., Kasper, M., Close, L. M., Brandner, W., \& Kellner, S. 2006, ApJ, 641, L141

Burgasser, A. J. 2007, ApJ, 659, 655

Burgasser, A. J., Cruz, K. L., Cushing, M., et al. 2010a, ApJ, 710, 1142 Burgasser, A. J., Cushing, M. C., Kirkpatrick, J. D., et al. 2011, ApJ, 735, 116 Burgasser, A. J., Geballe, T. R., Leggett, S. K., Kirkpatrick, J. D., \& Golimowski, D. A. 2006a, ApJ, 637, 1067

Burgasser, A. J., Kirkpatrick, J. D., Cruz, K. L., et al. 2006b, ApJS, 166, 585 Burgasser, A. J., Kirkpatrick, J. D., Cutri, R. M., et al. 2000, ApJ, 531, L57 Burgasser, A. J., Kirkpatrick, J. D., \& Lowrance, P. J. 2005, AJ, 129, 2849 Burgasser, A. J., McElwain, M. W., Kirkpatrick, J. D., et al. 2004, AJ, 127, 2856 Burgasser, A. J., Simcoe, R. A., Bochanski, J. J., et al. 2010b, ApJ, 725, 1405 Burningham, B., Pinfield, D. J., Leggett, S. K., et al. 2009, MNRAS, 395, 1237 Burrows, A., Marley, M., Hubbard, W. B., et al. 1997, ApJ, 491, 856 Casagrande, L., Schönrich, R., Asplund, M., et al. 2011, A\&A, 530, 138 Chabrier, G., \& Baraffe, I. 2000, ARA\&A, 38, 337

Cruz, K. L., Reid, I. N., Kirkpatrick, J. D., et al. 2007, AJ, 133, 439 Cushing, M. C., Marley, M. S., Saumon, D., et al. 2008, ApJ, 678, 1372 Cushing, M. C., Vacca, W. D., \& Rayner, J. T. 2004, PASP, 116, 362 Cushing, M. C., et al. 2011, ApJ, submitted

Day-Jones, A. C., Pinfield, D. J., Ruiz, M. T., et al. 2011, MNRAS, 410, 705 Dupuy, T. J., Liu, M. C., \& Ireland, M. J. 2009, ApJ, 699, 168

Faherty, J. K., Burgasser, A. J., Bochanski, J. J., et al. 2011, AJ, 141, 71 Faherty, J. K., Burgasser, A. J., Cruz, K. L., et al. 2009, AJ, 137, 1

Faherty, J. K., Burgasser, A. J., West, A. A., et al. 2010, AJ, 139, 176 Gizis, J. E., Troup, N. W., \& Burgasser, A. J. 2011, ApJ, 736, L34

Goldman, B., Marsat, S., Henning, T., Clemens, C., \& Greiner, J. 2010, MNRAS, 405, 1140

Golimowski, D. A., Leggett, S. K., Marley, M. S., et al. 2004, AJ, 127, 3516

Helling, C., Matthias, D., Woitke, P., \& Hauschildt, P. H. 2008, ApJ, 675, L105 Herter, T. L., et al. 2008, Proc. SPIE, 7014, 70140X

Holmberg, J., Nordström, B., \& Anderson, J. 2007, A\&A, 475, 519

Kirkpatrick, J. D. 2005, ARA\&A, 43, 195

Kirkpatrick, J. D., Dahn, C. C., Monet, D. G., et al. 2001, AJ, 127, 3235

Kirkpatrick, J. D., et al. 2011, ApJS, submitted

Lawrence, A., Warren, S. J., Almaini, O., et al. 2007, MNRAS, 379, 1599

Leggett, S. K., Burningham, B., Saumon, D., et al. 2010, ApJ, 710, 1627

Lepine, S. 2005, AJ, 130, 1680

Lepine, S. 2008, AJ, 135, 2177

Liu, M. C., Leggett, S. K., Golimowski, D. A., et al. 2006, ApJ, 647, 1393

Looper, D. L., Kirkpatrick, J. D., \& Burgasser, A. J. 2007, AJ, 134, 1162

Lowrance, P. J., Becklin, E. E., Schneider, G., et al. 2005, AJ, 130, 1845

Luhman, K. L., Burgasser, A. J., \& Bochanski, J. J. 2011, ApJ, 730, L9

Luhman, K. L., Patten, B. M., Marengo, M., et al. 2007, ApJ, 654, 570

Mainzer, A., Cushing, M. C., Skrutskie, M., et al. 2011, ApJ, 726, 30

Metchev, S., \& Hillenbrand, L. 2006, ApJ, 651, 1166

Mugrauer, M., Seifahrt, A., Neuhäuser, R., \& Mazeh, T. 2006, MNRAS, 373, L31

Nakajima, T., Oppenheimer, B. R., Kulkarni, S. R., et al. 1995, Nature, 378, 463

Nordström, B., Mayor, M., Andersen, J., et al. 2004, A\&A, 418, 989

Potter, D., Martin, E. L., Cushing, M. C., et al. 2002, ApJ, 567, L133

Radigan, J., Lafreniére, D., Jayawardhana, R., \& Doyon, R. 2008, ApJ, 689, 471

Reipurth, B., \& Clarke, C. 2001, AJ, 122, 432

Scholz, R.-D. 2010, A\&A, 515, A92

Scholz, R.-D., Bihain, G., Schnurr, O., \& Storm, J. 2011, A\&A, 532, L5

Scholz, R.-D., McCaughrean, M. J., Lodieu, N., \& Kuhlbrodt, B. 2003, A\&A, 398, L29

Skrutskie, M., Cutri, R. M., Stiening, R., et al. 2006, AJ, 131, 1163

Stamatellos, D., \& Whitworth, A. P. 2009, MNRAS, 392, 413 
Stephens, D. C., \& Leggett, S. K. 2004, PASP, 116, 9

Subasavage, J. P., Henry, T. J., Hambly, N. C., et al. 2005, AJ, 130, 1658

Vacca, W. D., Cushing, M. C., \& Rayner, J. T. 2003, PASP, 115, 389

van Leeuwen, F. 2007, A\&A, 474, 653
Vrba, F. J., Henden, A. A., Luginbuhl, C. B., et al. 2004, AJ, 127, 2948

Wilson, J. C., Kirkpatrick, J. D., Gizis, J. E., et al. 2001, AJ, 122, 1989

Wright, E. L., Eisenhardt, P. R. M., Mainzer, A. K., et al. 2010, AJ, 140, 1868

York, D. G., Adelman, J., Anderson, J. E., Jr., et al. 2000, AJ, 120, 1579

Zhang, Z. H., Pinfield, D. J., Day-Jones, A. C., et al. 2010, MNRAS, 404, 1817 